\title{
DIFFUSE GAMMA-RAY EMISSION FROM THE STARBURST GALAXY NGC 253
}

\author{
Timothy A. D. Paglione, Alan P. Marscher, and James M. Jackson \\ Department of Astronomy, Boston University, 725 Commonwealth Avenue, Boston, MA 02215; \\ paglione@fsh.bu.edu, marscher@buast0.bu.edu, jackson@slime.bu.edu \\ AND
}

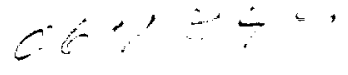

DAVID L. BERTSCH

NASA/Goddard Space Flight Center, Greenbelt, MD 20771; dlb@sage0.gsfc.nasa.gov

Received 1995 March 28 ; accepted 1995 September 26

\begin{abstract}
The starburst galaxy NGC 253 was observed with the Energetic Gamma Ray Experiment Telescope (EGRET) aboard the Compton Gamma Ray Observatory (CGRO) satellite. We obtain a $2 \sigma$ upper limit to the $\gamma$-ray emission above $100 \mathrm{MeV}$ of $8 \times 10^{-8}$ photons $\mathrm{cm}^{-2} \mathrm{~s}^{-1}$. Because of their large gas column densities and supernova rates, nearby starburst galaxies were predicted to have $\gamma$-ray fluxes detectable by EGRET. Our nondetection of $\gamma$-rays from NGC 253 motivates us to reexamine in detail the premise of supernova acceleration of cosmic rays and the effect of enhanced cloud densities, photon densities, and magnetic fields on the high-energy spectra of galaxies. By modeling the expected $\gamma$-ray and synchrotron spectra from NGC 253 , we find that up to $20 \%$ of the energy from supernovae is transferred to cosmic rays in the starburst, which is consistent with supernova acceleration models. Our calculations match the EGRET and radio data well with a supernova rate of $0.08 \mathrm{yr}^{-1}$, a magnetic field $B \gtrsim 5 \times 10^{-5} \mathrm{G}$, a density $n \sim 300 \mathrm{~cm}^{-3}$, a photon density $U_{\mathrm{ph}} \sim 200 \mathrm{eV} \mathrm{cm}^{-3}$, and an escape timescale $\tau_{0} \lesssim 10 \mathrm{Myr}$.
\end{abstract}

Subject headings: cosmic rays - galaxies: individual (NGC 253) -- galaxies: starburst -

gamma rays: observations - gamma rays: theory - stars: statistics

\section{INTRODUCTION}

\subsection{Diffuse Gamma-Ray Emission in Spiral Galaxies}

Since the earliest high-energy experiments, the diffuse $\gamma$-ray emission in the Milky Way has been shown to correlate closely with gas column density (see review by Bloemen 1989). In the interstellar medium (ISM), high-energy $\gamma$-rays $(E>100 \mathrm{MeV})$ are produced mostly through interactions between matter and cosmic-ray (CR) nuclei (inelastic collisions), and from electrons (bremsstrahlung emission and inverse Compton scattering). Cosmic rays are thought to originate in the shock fronts of supernova explosions (e.g., Ginzburg \& Syrovatskii 1964). Therefore, the $\gamma$-ray flux is a measure of the supernova rate and the average gas column density encountered by a CR before it escapes a galaxy. Hence, galaxies with high supernova rates and large gas masses should have more high-energy emission.

Of all galaxies, starburst galaxies have the highest inferred supernova rates and are predicted to have CR densities several hundred times larger than the Milky Way (Suchkov, Allen, \& Heckman 1993). The large far-infrared (FIR) luminosity of a starburst galaxy implies that the rate of massive star formation is very high, since this emission is presumed to be starlight reradiated by dust (Rieke et al. 1980). Starbursts also contain large masses of molecular gas as traced by $\mathrm{CO}$ (Young et al. 1989), and a significant fraction of that gas is dense, with $n_{\mathrm{H}_{2}}>10^{4} \mathrm{~cm}^{-3}$ (Paglione et al. 1995; Jackson et al. 1995; Helfer \& Blitz 1993; Wild et al. 1992; Sage, Shore, \& Solomon 1990; Carlstrom 1989; Mauersberger \& Henkel 1989).

\subsection{The Starburst Galaxy NGC 253}

NGC 253 is one of the archetypal starburst galaxies identified by Rieke et al. (1980). A supernova rate of roughly 0.1 $u c^{-1}$ has been derived from high-resolution radio continmees, which show many nonthermal point sources Uyestad 1988). From CO observations, the gas column density of the inner kiloparsec of NGC 253 is estimated to be $\sim 10^{23} \mathrm{~cm}^{-2}$ (Canzian, Mundy, \& Scoville 1988), roughly 10 times that of the Milky Way (e.g., Sanders, Solomon, \& Scoville 1984). From multiwavelength molecular line studies, the average density of the molecular clouds in NGC 253 is roughly $10^{5} \mathrm{~cm}^{-3}$, with a volume filling factor of $<10^{-3}$ (Paglione et al. 1995; Jackson et al. 1995; Wall et al. 1991; Mauersberger \& Henkel 1989). The average gas density over the starburst volume is therefore $30-300 \mathrm{~cm}^{-3}$, up to 100 times that of the Milky Way. (Throughout this paper we assume that the distance to NGC 253 is $3.4 \mathrm{Mpc}$.)

The continuum spectrum of NGC 253 peaks in the FIR at $\sim 100 \mu \mathrm{m}$ with a luminosity of $L_{\mathrm{FIR}}=3 \times 10^{10} L_{\odot}$ (Rice et al. 1988), roughly 5 times the FIR luminosity of the Milky Way (Cox \& Mezger 1989). Because the IR region is concentrated within the central $500 \mathrm{pc}$ of the starburst nucleus (Piña et al. 1992; Heckman, Armus, \& Miley 1990), the photon density in this region of NGC 253 is much larger than in the Milky Way. Therefore, inverse Compton scattering of the FIR radiation off the large population of CR electrons in NGC 253 (Carilli et al. 1992) should be significant (Goldshmidt \& Rephaeli 1995).

Another well-known starburst galaxy, M82, which has similar gas properties and nearly the same supernova rate as NGC 253 , may have a CR density roughly 300 times higher than that of the Milky Way (Suchkov et al. 1993). Because the $\gamma$-ray flux from M82 was predicted to be detectable with EGRET (Akyüz, Brouillet, \& Özel 1991), we anticipated that NGC 253 would be detectable in hard $\gamma$-rays as well. However, preliminary EGRET results show only upper limits to the $\gamma$-ray emission from M82 and NGC 253 (Sreekumar et al. 1994). Here we analyze EGRET observations of $\gamma$-ray emission from NGC 253 and examine in detail the high-energy flux expected from a starburst galaxy. 


\section{OBSERVATIONS AND RESULTS}

The EGRET instrument is sensitive to photons in the energy range from roughly $20 \mathrm{MeV}$ to $30 \mathrm{GeV}$. A complete description of EGRET is given by Hughes et al. (1980) and Kanbach et al. (1988, 1989). Thompson et al. (1993) present the results of the instrument calibration.

NGC 253 was observed for a total on-axis integration time of roughly 2 weeks. We subtracted a background modeled after the Galactic $\mathrm{H}$ I emission. This measurement is very sensitive, since NGC 253 is located at the south Galactic pole where the background is minimal. We thereby obtain a $2 \sigma$ upper limit of $8 \times 10^{-8}$ photons $\mathrm{cm}^{-2} \mathrm{~s}^{-1}$ for the flux above $100 \mathrm{MeV}$ from NGC 253. This limit we find from reanalyzing the EGRET data is slightly different from, yet consistent with, that found by Sreekumar et al. (1994).

\section{THE COSMIC-RAY ELECTRON DISTRIBUTION IN CLOUDS}

The lack of significant $\gamma$-ray emission from M82 is consistent with the predictions of Akyüz et al. (1991) and Pohl (1994), given the sensitivity of EGRET. However, this result does not immediately explain our nondetection from NGC 253 since the smaller size, higher average molecular gas density, and younger age of the starburst in NGC 253 imply that the CR density $\left(n_{\mathrm{CR}}\right)$ and $\gamma$-ray emissivity of NGC 253 should be higher than that of M82. The starburst volume of NGC 253 is smaller than that of M82 in radio, infrared, and CO maps (Piña et al. 1992; Telesco et al. 1991; Carlstrom et al. 1989; Canzian et al. 1988), which indicates not only an increase in the CR density in NGC 253 despite a lower supernova rate, but also an increased photon density, which yields a higher inverse Compton flux. The larger average molecular gas density in NGC 253 (Jackson et al. 1995) implies higher secondary electron and neutral pion production, which in turn lead to enhanced $\gamma$-ray emission. Rieke, Lebofski, \& Walker (1988) postulated that the starburst in NGC 253 is less evolved than that of M82. Also, radio spectral index maps imply that diffusion rather than convection is still a major escape mechanism for CR electrons in NGC 253 (Carilli et al. 1992). Therefore, cosmic rays should remain near molecular clouds longer in NGC 253 than in M82 (see Seaquist \& Odegard 1991) and thereby support reactions that lead to $\gamma$-ray emission. Finally, several other independent methods for estimating $n_{\mathrm{CR}}$ in starburst galaxies suggest that the CR density is higher in NGC 253 than in M82 by factors of a few (Suchkov et al. 1993).

Therefore, we proceed to calculate the expected $\gamma$-ray flux from NGC 253 based on the observed supernova rate, gas density, and synchrotron radio emission.

\subsection{Modeling the Interstellar Cosmic-Ray Distribution}

To calculate the CR density and $\gamma$-ray flux from a starburst, we model the $C R$ production and radiation mechanisms from a region with dense clouds and a high supernova rate. An initial CR spectrum must be assumed, and the energy distribution of CR electrons must be generated. We consider the contribution of both primary and secondary electrons, since secondary production in the dense environment of a starburst nucleus is probably significant (Marscher \& Brown 1978). We also examine the effects of a large photon density, magnetic field, and cloud density on the CR energy distribution and subsequent $\gamma$-ray emissivity.

In $\S 3.2$ we outline the procedure for generating the steady state CR energy distribution in the ISM. The discussion concentrates on secondary electron production and loss mechanisms. We discuss the final form of the secondary electron distribution in $\S 3.3$, and we describe the primary CR electron spectrum in $\S 3.5$. We determine the input $C R$ proton spectrum in $\S 3.4$. We discuss the consequent radio and $\gamma$-ray emission in $\S 4$.

\subsection{Solution to the Diffusion-Loss Equation}

The procedure we follow for generating the secondary electron spectrum of an interstellar cloud was outlined by Marscher \& Brown (1978). The general steady state diffusion-loss equation for CR electrons may be written as (e.g., Longair 1981)

$$
\frac{N(E)}{\tau(E)}-\frac{d}{d E}[b(E) N(E)]-Q(E)=0 .
$$

The electron energy distribution (the number of electrons with energy between $E$ and $E+d E$ ) is $N(E)$. The source function $Q(E)$ is the sum of the production rates of secondary electrons. The total time-independent loss rate is $1 / \tau(E)$. The electron energy loss rate, $b(E)=-d E / d t$, is given by (e.g., Ginzburg \& Syrovatskii 1964)

$$
\begin{aligned}
b(E)= & 2.52 \times 10^{-17} n\left[6.27+\ln \left(E / m c^{2}\right)\right]+(V / R) E \\
& +1.02 \times 10^{-16}\left[U_{\mathrm{ph}}+2.49 \times 10^{10} B^{2}\right] E^{2} \mathrm{GeV} \mathrm{s}^{-1}
\end{aligned}
$$

Ionization losses, which are important at lower energies, are represented in the first term of equation (2), where $n$ is the average interstellar density, $E$ is the particle energy, and $m c^{2}$ is the electron rest energy. The second term is the loss rate due to adiabatic expansion. For NGC 253, the starburst wind speed is $V \sim 300 \mathrm{~km} \mathrm{~s}^{-1}$ and the starburst radius is $R=325$ pc (Heckman et al. 1990; Canzian et al. 1988; McCarthy, Heckman, \& van Breugel 1987). Adiabatic expansion losses have a minor effect on the electron distribution and may be treated as an effective sink. The last term is the loss rate due to synchrotron radiation and inverse Compton scattering, where the photon energy density $U_{\mathrm{ph}}$ is in $\mathrm{eV} \mathrm{cm} \mathrm{cm}^{-3}$, and the magnetic field $B$ is in gauss.

We assume that the change in the electron distribution due to bremsstrahlung radiation may be treated with a single characteristic loss rate $1 / \tau_{B}$, and that an electron loses all its energy in the interaction. The total time-independent loss rate is comprised of the energy-dependent diffusion rate and the bremsstrahlung loss rate (Blandford \& Ostriker 1980; Ormes \& Freier 1978),

$$
\tau^{1}(E)=\tau_{B}^{-1}(E)+\tau_{D}{ }^{1}(E)
$$

where

$$
\tau_{B}^{-1}(E)=9.6 \times 10^{16} n \mathrm{~s}^{1}
$$

and

$$
\tau_{\mathrm{D}}^{-1}(E)=\tau_{0}^{-1} \beta E_{\mathrm{GeV}}^{0.5}+\tau_{c} .
$$

The characteristic escape time in a "leaky box" model is $\tau_{D}$, where $\beta$ is the particle velocity in light units, and $\tau_{c}$ is the convection timescale. We assume that the convection-loss 
timescale is within an order of magnitude of the energydependent escape time $\tau_{0}$ at $1 \mathrm{GeV}$ based on the evidence of supernova winds in the nucleus of NGC 253 (McCarthy et al. 1987; Antonucci \& Ulvestad 1988) and convective "chimneys" in its disk (Carilli et al. 1992). Our calculations include the cases $\tau_{0}=0.1,1$, and $10 \mathrm{Myr}$, and we assume $\tau_{c} \approx 1$ Myr.

Equation (1) may be solved analytically with the integrating factor

$$
b(E) \exp \left[-\int_{E_{0}}^{E} \frac{d E^{\prime}}{\tau\left(E^{\prime}\right) b\left(E^{\prime}\right)}\right] .
$$

The equation for the electron energy spectrum in a cloud is then

$$
N(E)=\int_{t_{0}}^{t} Q\left(E^{\prime}\right)\left|\frac{\partial E^{\prime}}{\partial E}\right| \exp \left[-F\left(E^{\prime}, E\right)\right] d t^{\prime},
$$

where $F\left(E^{\prime}, E\right)$ is defined as the integral in equation (4).

\subsection{Secondary Electron Production}

The three reactions between CRs and interstellar matter that produce most of the interstellar secondary electrons are knock-on collisions, neutron beta decays, and charged-pion decays. Each source term can be written as

$$
Q_{i}(E) \propto n \int \frac{d \sigma\left(E, E_{p}\right)}{d E_{p}} \frac{d J_{p}}{d E_{p}} d E_{p},
$$

where $d J_{p} / d E_{p}=N_{p}\left(E_{p}\right) \beta c / 4 \pi$ is the differential energy spectrum of $\mathrm{CR}$ protons, and $d \sigma\left(E, E_{p}\right) / d E_{p}$ is the differential cross section for the interaction. The $\mathrm{CR}$ proton spectrum $N_{p}$ will be discussed in $\$ 3.4$.

Knock-on or Coulomb collisions produce CR electrons when a high-energy particle transfers energy much greater than the binding energy to an atomic electron. We use the form of the knock-on cross section given by Abraham, Brunstein, \& Cline (1966). The resulting spectrum of secondary electrons follows an approximately constant power law that is up to $20 \%$ steeper than the original proton injection spectrum. The secondary electron spectrum from neutron beta decays is roughly an order of magnitude lower than that from knock-on collisions and has a similar slope (Abraham et al. 1966).

The above two mechanisms produce most of the lower energy CR secondaries. Charged-pion decay $\left(\pi^{ \pm} \rightarrow \mu^{ \pm} \rightarrow e^{ \pm}\right)$dominates the production of higher energy CR electrons $(E \gtrsim 100 \mathrm{MeV}$ ). Dermer (1986) provides a concise overview and comparison of past studies of pion production in the ISM. We follow the procedure outlined by Marscher \& Brown (1978). The pion and subsequent electron source spectra peak below $100 \mathrm{MeV}\left(\approx \frac{1}{2} m_{\pi} c^{2}\right)$, and the slope approaches that of the incident $C R$ proton spectrum above $1 \mathrm{GeV}$ (see Dermer 1986).

The total secondary electron distribution is the summation of the above source spectra. The result is displayed in Figure 1. The steady state secondary CR electron distribution calculated from the total source spectrum with equation (5) is shown in Figure 2. The high-energy electron spectrum is steeper for an increased magnetic field and photon field owing to enhanced synchrotron and inverse Compton losses. Bremsstrahlung and adiabatic expansion losses decrease the entire spectrum while maintaining the shape and are most pronounced, yet still a minor effect, near $1 \mathrm{GeV}$. A shorter characteristic escape time decreases the high-energy $C R$ spectrum but does not change the slope. Escape losses have a stronger effect on the total production rate of secondary electrons, since these losses have a stronger bearing on the steady state CR proton distribution (see $\S 3.4)$.

\subsection{Cosmic-Ray Proton Energy Spectrum}

The proton energy distribution is required to derive the secondary electron source functions in $\$ 3.3$. The proton spectrum is usually assumed to follow a power law proportional to $E_{p}{ }^{s}$, where $s=2.52 .75$ approximates the local CR distribution (Dermer 1986). However, because the goal of this project is to test the mechanism of supernova acceleration of cosmic rays, we constrain only the $C R$ injection spectrum, and we assume that it is constant with time. The

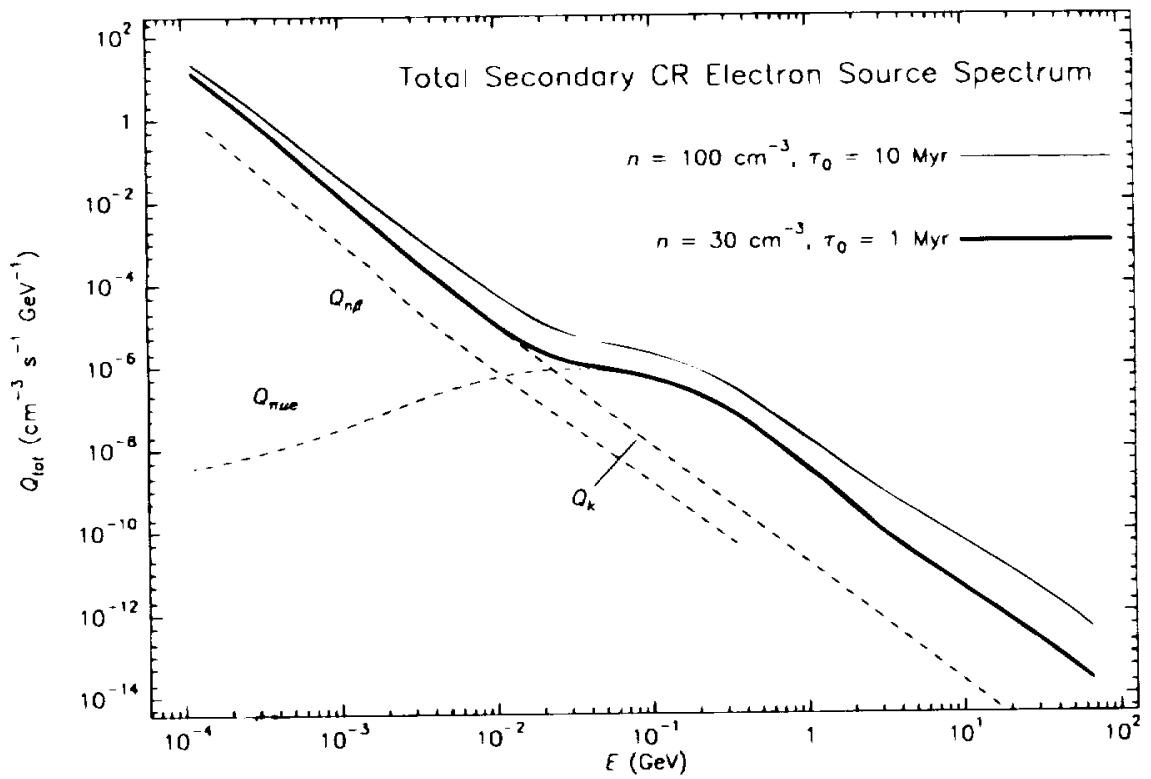

Fig. 1. The total secondary CR electron source spectrum (heavy line) from knock-on $\left(Q_{k}\right)$, neutron beta decay $\left(Q_{n \beta}\right)$, and pion-muon-electron decay $\left(Q_{\pi \mu e}\right)$ reactions (dashed lines) for two values of the density $n$ and escape time $\tau_{0}$ in a "leaky box" model of diffusion. 


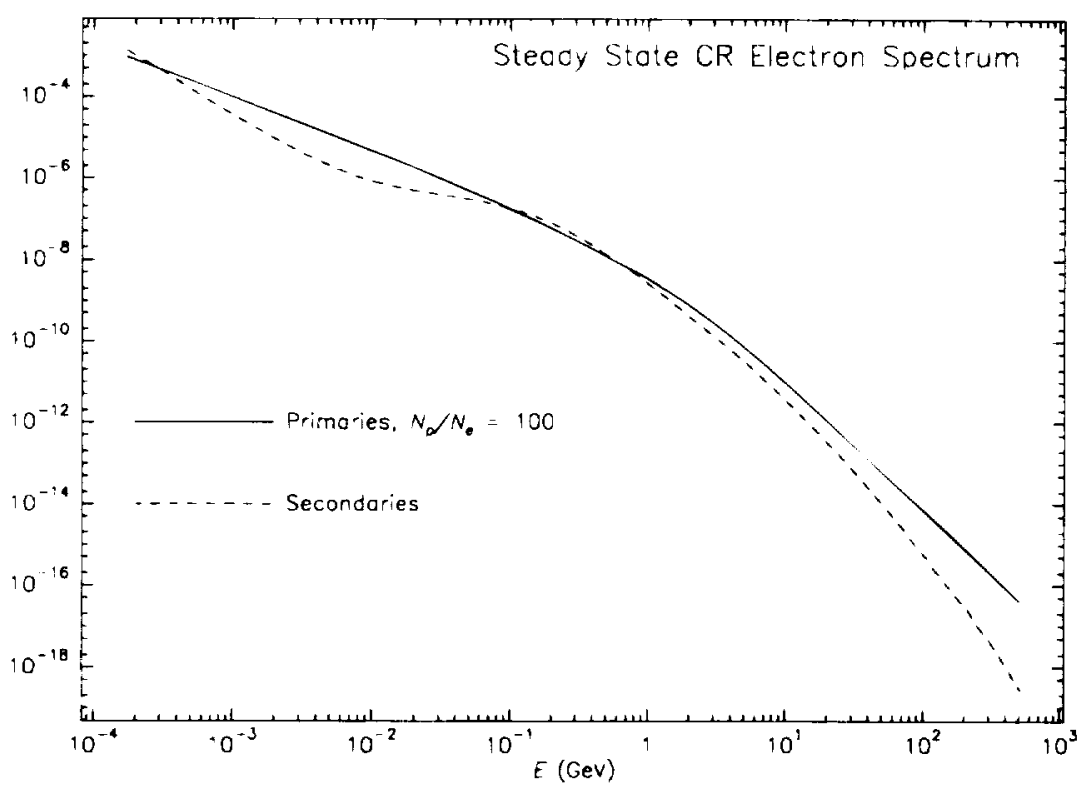

FIG. 2. The steady state primary CR electron spectrum (continuous line) compared with that of secondary electrons (dashed line) for $n=100 \mathrm{~cm}^{-3}$, $B=5 \times 10^{5} \mathrm{G}, U_{\mathrm{ph}}=200 \mathrm{eV} \mathrm{cm}^{-3}, \tau_{0}=10 \mathrm{Myr}$, and $N_{p} / N_{\mathrm{e}}=100$

initial proton spectrum $N_{p}\left(E_{p}\right)$ takes the form of equation (5) with an injection spectrum $Q \propto E_{p}^{-s}$. This CR distribution changes with time owing to ionization and escape losses as the cosmic rays encounter the ISM. The loss time $\tau\left(E_{p}\right)$ is now given only by $\tau_{D}$ in equation (3), and the energy loss rate is (e.g., Ginzburg \& Syrovatskii 1964)

$$
\begin{aligned}
b\left(E_{p}\right)= & 1.84 \times 10^{-17} n \frac{c}{v}\left[11.1+2 \ln \gamma_{p}+\ln \left(\frac{v}{c}\right)^{2}-\left(\frac{v}{c}\right)^{2}\right] \\
& +0.65 n c \sigma_{n} E_{p} \mathrm{GeV} \mathrm{s}^{-1},
\end{aligned}
$$

where $v$ is the particle velocity, and $\gamma_{p}$ is the total proton energy divided by the proton rest energy $M c^{2}$. For protons with total energy $\geq 1.22 \mathrm{GeV}$, losses due to pion production must also be included. For this term, we use the relation given by Mannheim \& Schlickeiser (1994), where $\sigma_{\pi}=3$ $\times 10^{-26} \mathrm{~cm}^{2}$ is the total pion cross section, and $E_{p}$ $+M c^{2} \geq 1.22 \mathrm{GeV}$.

The value of the spectral index $s$ is estimated from previous observations of Galactic and extragalactic supernovae (Reynolds \& Ellison 1992; Huang et al. 1994), as well as the local Galactic CR distribution (e.g., Ormes \& Freier 1978 ) and the nucleus of M82 (Pohl 1994). Indices between 2 and 2.6 are observed for most supernova remnants and are predicted from blast-wave theory and models of firstorder Fermi acceleration of CRs (Bell 1978a, b; Reynolds \& Ellison 1992, and references within). We consider two models with $s=2.2$ and 2.5 .

The calculated steady state proton spectrum is shown in Figure 3. The distribution with $s=2.2$ compares well with

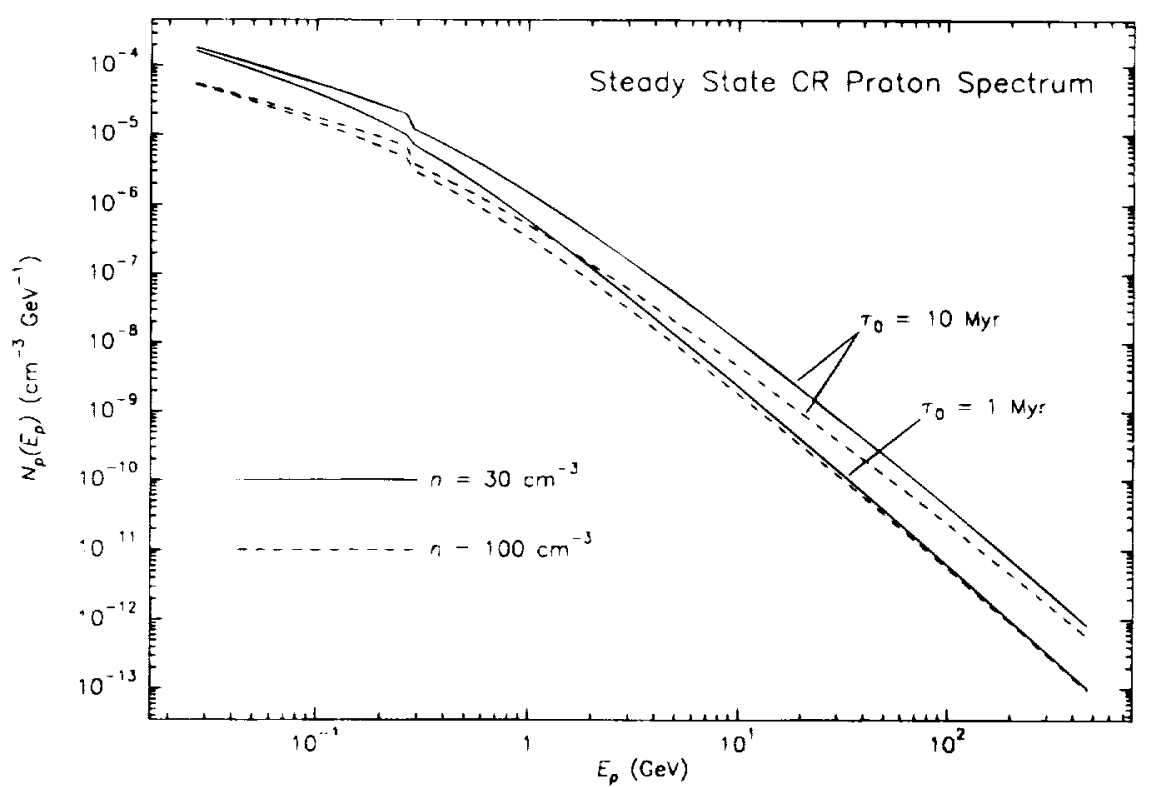

FIG. 3.- - The steady state CR proton spectrum for two values of the density and escape timescale. The kink just below $0.3 \mathrm{GeV}$ results from the threshold to pion production. 
that of the Milky Way (see Dermer 1986; Ormes \& Freier 1978; Bell 1978a, b). Note that the spectrum decreases with density at lower energies because of ionization losses. The high-energy protons reach a steady state by either the characteristic escape time $\tau_{0}$, or the time for energy loss from pion production $\tau_{\pi} \sim 0.5(\mathrm{n} / 100 \mathrm{~cm})^{-3} \mathrm{Myr}$. Therefore at higher densities, pion production dominates the energy losses above $1 \mathrm{GeV}$, but for lower densities, the pion production and escape loss rates are comparable (Fig. 3).

\subsection{Primary Electron Distribution}

The contribution of primary CR electrons to the diffuse $\gamma$-ray emission in NGC 253 must also be determined. Primary electrons are presumably accelerated in supernova explosions in the same way as protons; the injection spectrum is a power law with $s=2-2.6$. Figure 2 shows the steady state primary electron distribution calculated from equation (5).

\subsection{Normalization of the $C R$ Injection Spectrum}

In $\$ 3.4$ we introduced the CR proton injection spectrum as $Q=K E^{-s}$. The constant of proportionality $K$ may be obtained from the total power transferred by supernovae into cosmic rays within a given volume

$$
\int_{E_{\min }}^{\infty} Q E d E=\eta P \Psi / \mathscr{V}
$$

Here $\eta P$ is the energy transferred from a supernova to CRs, where $P \approx 10^{51}$ ergs, and $\eta$ is the transfer efficiency. The supernova rate is $\Psi$, and $\mathscr{V}$ is the volume. Solving for the normalization, we find (for $s>2$ )

$$
K=\frac{\eta P \Psi}{\mathscr{V}}(s-2) E_{\min }^{s-2} .
$$

We treat the case of $s=2.2$ and $E_{\min }=100 \mathrm{MeV}$.

An additional scaling factor, the proton-to-electron flux ratio $N_{p} / N_{e}$, must be included for primary electrons. This ratio is 100 for the Milky Way, but it could be smaller if CRs are accelerated by multiple shocks (Bell 1978b). X-ray data for M82 are consistent with $N_{p} / N_{e}$ as low as 30 , although a ratio of 100 , which implies a lower energy transfer efficiency, may be more appropriate (Völk, Klien, \& Wielebinski 1989a).

\section{DISCUSSION}

We use the calculations of $\S 3$ to model the radio and $\gamma$-ray emission from the starburst galaxy NGC 253. The starburst nucleus is assumed to be a uniform cylinder with a constant supernova rate. We have examined the expected emission from the disk of NGC 253 as well, where the source of CRs is the population leaving the nuclear region. However, we find the high-energy emission from the disk to be negligible, and the parameters needed for the calculations are poorly constrained by observations. Therefore, we approximate that all the $\gamma$-ray emission from NGC 253 originates in the nucleus, and once a cosmic ray leaves the starburst region, it is no longer considered in the model.

\subsection{Radio Emission}

Radio images of NGC 253 show a prominent nucleus which matches the dimensions of the $\mathrm{CO}$ and IR starburst regions (Piña et al. 1992; Antonucci \& Ulvestad 1988;
Canzian et al. 1988). The spectrum from the nucleus is flat (Ulvestad \& Antonucci 1991), and there is also considerable nonthermal emission within the disk and in a large, steeper spectrum, diffuse halo around the galaxy (Beck et al. 1994; Carilli et al. 1992; Klein et al. 1983; Beck et al. 1979).

We calculate the synchrotron emissivity $\epsilon_{v}$ with the electron distribution from $\S 3$ assuming an isotropic distribution of pitch angles. We include thermal free-free emission $j_{v}^{f f}$ with $T=8000 \mathrm{~K}$, as well as free-free absorption and dust emission in order to determine the total continuum flux density of NGC 253. We vary the emission measure EM so that the thermal flux density matches millimeter observations (Carlstrom et al. 1989; Elias et al. 1978). The total radio continuum flux density is then given by

$$
S_{v}=f_{v}^{f f} \Omega+\frac{\epsilon_{v}}{\tau_{v}^{f f}}\left(1-e^{\tau_{v} \mathrm{ff}^{\mathrm{ff}}}\right) \Omega l,
$$

where the free-free optical depth $\tau_{v}^{\text {ff }} \propto$ EM. The source solid angle $\Omega \sim 300$ arcsec $^{2}$ and the line-of-sight depth $l \sim 630 \mathrm{pc}$ are estimated from $\mathrm{CO}$ interferometry and $6 \mathrm{~cm}$ images of NGC 253 (Canzian et al. 1988; Antonucci \& Ulvestad 1988). We also calculate the dust emission assuming $S_{v}$ (dust) $\propto v^{1.5} B_{v}\left(T_{D}\right)$, where $B_{v}$ is the Planck function and $T_{D} \approx 40 \mathrm{~K}$ (Telesco \& Harper 1980).

For normalization, we choose a cylindrical starburst region $70 \mathrm{pc}$ thick, with a radius of $325 \mathrm{pc}$ (Canzian et al. 1988; Antonucci \& Ulvestad 1988), and $\Psi=0.08 \mathrm{yr}^{1}{ }^{1}$ (Van Buren \& Greenhouse 1994; Ulvestad \& Antonucci 1994). The total modeled radio and FIR spectrum from the nucleus of NGC 253 is shown in Figure 4. The model reproduces the radio spectrum of the nucleus of NGC 253 for the following values of the free parameters: $s=2.2, B \geq 5$ $\times 10^{5} \mathrm{G}, n=300 \mathrm{~cm}^{-3}, U_{\mathrm{ph}}=200 \mathrm{eV} \mathrm{cm}{ }^{3}, \tau_{0}=10$ Myr, and EM $\sim 10^{5} \mathrm{~cm}{ }^{6}$ pc.

If we consider only the emission from secondary electrons, the required efficiency of energy transfer from supernovae to CRs is roughly $50 \%$. Either CR acceleration in starburst galaxies is very efficient, or primary electrons contribute in part to the emission. To include emission from primary electrons, we must evaluate the proton-to-electron ratio, and the choice of this ratio affects the efficiency. Figure 5 shows how this ratio $N_{p} / N_{e}$ depends on the energy transfer efficiency, $\eta$. Also shown is the fraction of the total flux due to emission from secondaries $F_{2 \mathrm{dy}} / F_{\text {tot }}$. Given $N_{p} / N_{e}=100$, we find $\eta \sim 20 \%$, which is somewhat higher than values predicted for the Milky Way (see Volk et al. 1989a; Volk, Klien, \& Wielebinski 1989b; and references therein). In the case of a lower $N_{p} / N_{e}$ ratio of 30 , which is possible if CRs are shock accelerated more than once (Bell 1978 b), the efficiency necessary to match the radio emission is only $9 \%$. Note that the contribution from secondary electrons is significant in a starburst nucleus, whereas primary electrons dominate Galactic synchrotron emission (Marscher \& Brown 1978).

\subsection{Gamma-Ray Emission \\ 4.2.1. Cosmic-Ray Electrons}

Bremsstrahlung emission and inverse Compton scattering of CR electrons are prominent sources of soft $\gamma$-rays $(E<100 \mathrm{MeV})$. In the Galaxy, inverse Compton scattering is not so important (Bloemen 1989), but it may be in starburst galaxies because of their high photon densities. Similarly, the bremsstrahlung emission may be enhanced owing to the higher average densities. 


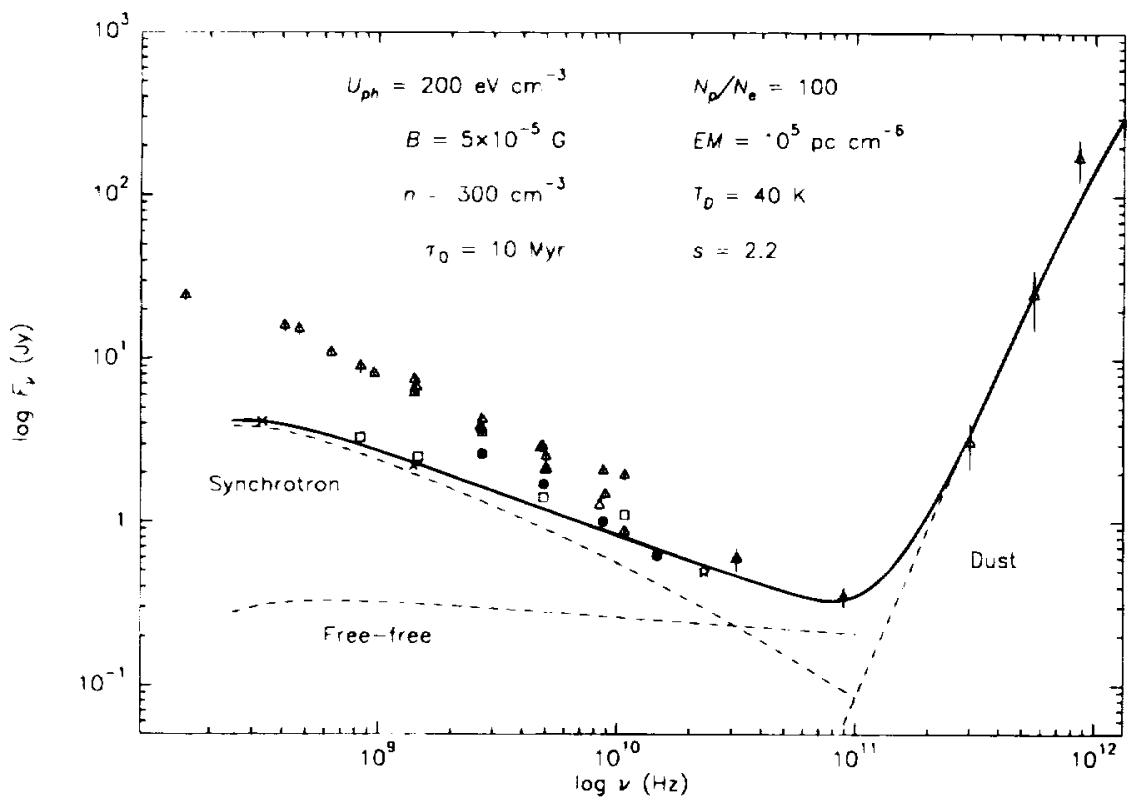

FIG. 4. - The radio and FIR spectrum of NGC 253. The triangles are the integrated flux densities (Carlstrom et al. 1989; Klein et al. 1983; Kühr et al. 1981; Telesco \& Harper 1980; Elias et al. 1978). The other symbols are the intensities from only the nucleus (crosses, Carilli et al. 1992; squares, Hummel, Smith, \& van der Hulst $1984 ;$ star, Klein \& Emerson 1981; dots, Beck et al. 1979). The model synchrotron spectrum is the summation of the primary and
secondary electron emission.

We generate the inverse Compton emissivity with the CR electron distribution calculated in $\S 3$ using well-known equations (Rybicki \& Lightman 1979 , p. 207). To model the emission from NGC 253, we again assume a photon energy density of $U_{\mathrm{ph}}=200 \mathrm{eV} \mathrm{cm}{ }^{-3}$ from the FIR luminosity (Rice et al. 1988) and the size of the starburst region (Antonucci \& Ulvestad 1988; Canzian et al. 1988; Piña et al. 1992). The bremsstrahlung emissivity is calculated from the $\mathrm{CR}$ electron spectrum with

$$
Q_{B}\left(E_{\gamma}\right)=n \sigma c E_{\gamma}^{-1} \int_{E_{\gamma}}^{\infty} N(E) d E \mathrm{~cm}^{-3} \mathrm{~s}^{-1} \mathrm{GeV}^{-1}
$$

In the Galaxy, the contribution of inverse Compton $\gamma$-rays to the total diffuse $\gamma$-ray emission is roughly $10 \%$ of that from bremsstrahlung (e.g., Bloemen 1989). In NGC 253 , this ratio is preserved because the average cloud density and photon field (and consequently, the bremsstrahlung and inverse Compton emission) are higher than in the Milky Way by roughly the same factor of $\sim 100$.

\subsubsection{Neutral Pion Decays}

The dominant mechanism for generating diffuse $\gamma$-ray emission $(E>100 \mathrm{MeV})$ in the ISM is neutral pion decays. The neutral pion spectrum is half the total charged pion spectrum discussed in $\S 3.3$. Each $\pi^{0}$ decays into two $\gamma$-rays. The source function of $\gamma$-rays from $\pi^{0}$ decays is (Cavallo \& Gould 1971)

$$
Q_{\pi^{0}}\left(E_{\gamma}\right)=2 \int_{E_{1}}^{\infty} \frac{(1 / 2) Q_{\pi}\left(E_{\pi}\right)}{\left(E_{\pi}^{2}-4 E_{0}^{2}\right)^{1 / 2}} d E_{\pi} \mathrm{cm}^{-3} \mathrm{~s}^{-1} \mathrm{GeV}^{-1}
$$

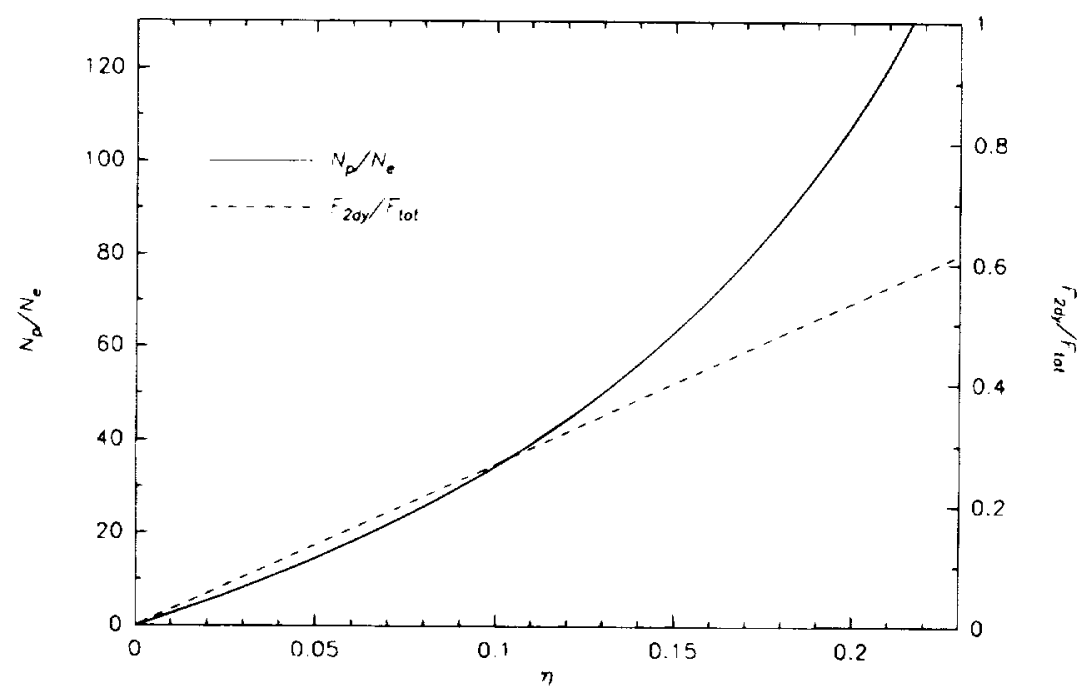

Fig. 5. The proton to electron ratio $N_{p} / N_{e}$ and the fraction of emission from secondary electrons $F_{2 \mathrm{dy}} / F_{\text {tol }}$, vs. the efficiency $\eta$ by which the total energy of a supernova is converted into cosmic rays. 


\subsection{The Expected Gamma-Ray Spectrum from a Spiral Galaxy 4.3.1. NGC 253}

The model $\gamma$-ray spectrum of NGC 253 is shown in Figure 6. Above $100 \mathrm{MeV}$, the emission is dominated by neutral pion decays, and the flux near $1 \mathrm{MeV}$ is due mostly to inverse Compton scattering. Our model greatly underestimates the lower energy $(0.05 \mathrm{MeV}<E<10 \mathrm{MeV}) \gamma$-ray flux seen from OSSE observations (Bhattacharya et al. 1994), but recent analysis of these data has shown that most of the emission arises not in the nucleus, but from the disk of NGC 253 (Goldshmidt \& Rephaeli 1995). Our inverse Compton flux is somewhat lower than that modeled for the nucleus by Goldshmidt \& Rephaeli (1995), but our results are not inconsistent with theirs considering the differences in our initial assumptions. The total integrated flux of $\gamma$-rays above $100 \mathrm{MeV}$ from Figure 6 is $4 \times 10^{-8}$ photons $\mathrm{cm}^{-2}$ $\mathrm{s}^{-1}$. Moderate changes in the free parameters can decrease the model $\gamma$-ray flux by up to a factor of 2 and still match the synchrotron spectrum. If we force our inverse Compton spectrum to match the OSSE data, the predicted flux above $100 \mathrm{MeV}$ is $5 \times 10^{8}$ photons $\mathrm{cm}^{-2} \mathrm{~s}^{-1}$, which is still consistent with the EGRET limit.

Typical age estimates for the starburst in NGC 253 are $\sim 10^{7}$ yr (Rieke et al. 1980, 1988). The CR distribution should reach a steady state in this time. However, if it has not, the evolving CR spectrum is slightly different than the ones shown in Figure 3. Because the CR protons below $1 \mathrm{GeV}$ reach a steady state much more quickly than those at high energies, the evolving distribution resembles one in steady state spectrum with a lower escape timescale (see Fig. 3). The resulting secondary electron and synchrotron spectra are slightly lower and flatter, and the $\pi^{\circ}$ decay $\gamma$-ray flux is lower as well. The radio spectrum of NGC 253 is matched best by a steady state CR distribution given $\tau_{0}=$ $10 \mathrm{Myr}$, and we conclude that the CRs have reached equilibrium.

\subsubsection{The Galaxy}

In comparison, the $\gamma$-ray flux from the Galaxy (Fichtel et al. 1993; Bloemen 1989) is matched well by the model if we assume a supernova rate of $1 / 50 \mathrm{yr}^{-1}$ (Tammann 1974) and a cylindrical volume $10 \mathrm{kpc}$ in radius and $250 \mathrm{pc}$ thick. The transfer efficiency necessary to match the $\operatorname{COS} B$ and EGRET data is $\eta \sim 5 \%$, in good agreement with theoretical calculations (Markiewicz, Drury, \& Völk 1990; Drury, Markiewicz, \& Völk 1989). We assume $B=10^{-6} \mathrm{G}, n=1$ $\mathrm{cm}^{3}, \tau_{0}=10 \mathrm{Myr}$, and $U_{\mathrm{ph}}=1 \mathrm{eV} \mathrm{cm}^{-3}$ in the Galaxy. Unlike the starburst galaxy, emission from primary electrons dominates the flux from the Milky Way, given $N_{p} / N_{e}=100$.

\subsection{3. $M 82$}

The synchrotron spectrum of M82 is flatter than that of NGC 253 (Klein, Wielebinski, \& Morsi 1988), and it is matched by the model if we adopt somewhat lower values for the magnetic field, photon field, and characteristic escape time than those used for NGC 253. These changes are supported by the arguments at the beginning of $\S 3$, and they result in a lower $\gamma$-ray flux from M82, consistent with the EGRET limit of $5 \times 10^{-8}$ photons $\mathrm{cm}^{-2} \mathrm{~s}^{-1}$ (Sreekumar et al. 1994) and other model calculations (Pohl 1994; Akyüz et al. 1991). We should mention that the flatter synchrotron spectrum is also consistent with higher densities owing to the decrease in low-energy CRs through ionization losses. However, molecular line studies of these galaxies indicate that the average densities of the cloud cores in M82 are up to 10 times lower than those in NGC 253 (Jackson et al. 1995), and higher densities also imply an increase in $\gamma$-ray flux. Therefore, the average gas densities in M82 are most likely lower than those in NGC 253, and the flatter synchrotron spectrum of M82 is a result of a lower magnetic field and photon density. We cannot exclude the possibility that the $C R$ production rates are different in these two galaxies either, since the physical conditions of the ISM in galaxies regulate both the production of $\gamma$-rays and CR losses. Thus, changing the density and photon field tends to have little effect on the high-energy emission (Pohl 1994).

\section{CONCLUSIONS}

We obtain a sensitive upper limit to the $\gamma$-ray flux from the starburst galaxy NGC 253 of $8 \times 10^{-8}$ photons $\mathrm{cm}^{-2}$

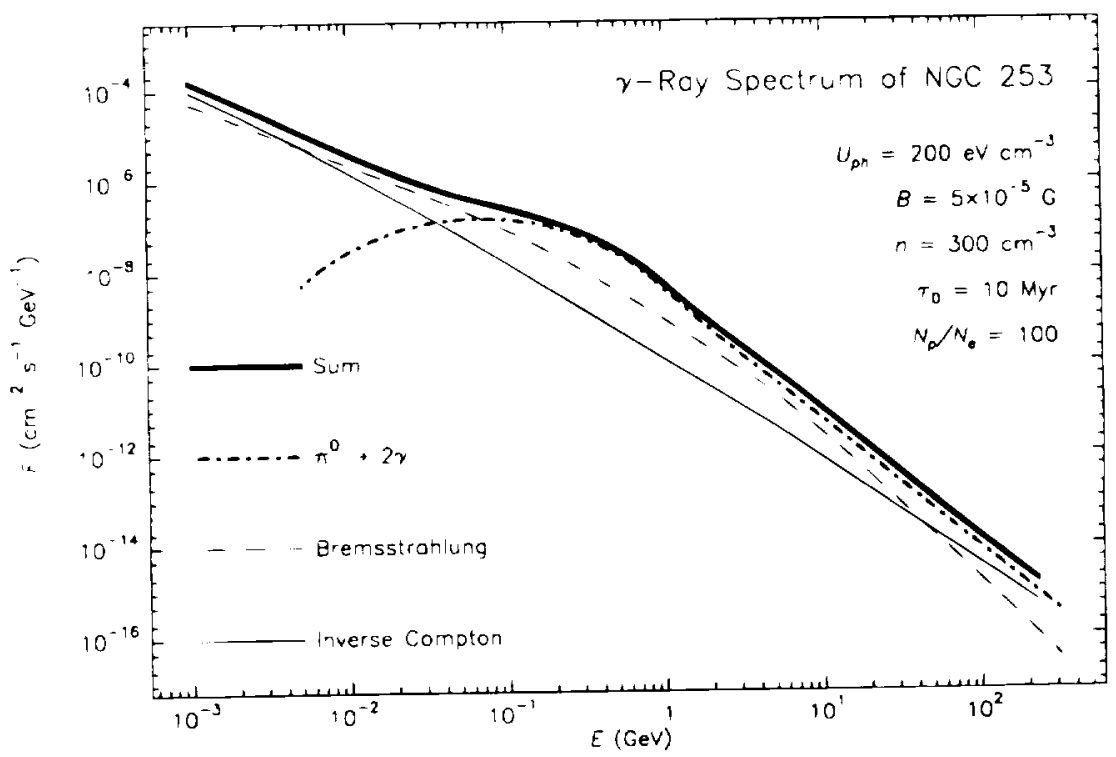

FiG. 6. The model $\gamma$-ray spectrum of NGC 253 from bremsstrablung, inverse Compton, and $\pi^{0}$ decays 
$\mathrm{s}^{-1}$ with EGRET. For comparison, we determine the steady state energy distribution of CRs in the ISM of a starburst galaxy. The model fits the synchrotron spectrum from NGC 253 well, given the high magnetic fields, gas densities, and photon fields in starburst nuclei. We derive a $\gamma$-ray flux from the nucleus of NGC 253 of $4 \times 10^{-8}$ photons $\mathrm{cm}^{-2} \mathrm{~s}^{-1}$. This result assumes a supernova rate of $0.08 \mathrm{yr}^{-1}$, a magnetic field $B \gtrsim 5 \times 10^{-5} \mathrm{G}$, an escape timescale $\tau_{0} \sim 10$ Myr, a density $n \sim 300 \mathrm{~cm}^{-3}$, and a photon density $U_{\mathrm{ph}} \sim$ $200 \mathrm{eV} \mathrm{cm}{ }^{-3}$. From these calculations, we find that up to $20 \%$ of the energy from supernovae in the starburst is transferred to CRs, which is somewhat larger that the value derived for the Galaxy $(3 \%-10 \%)$, unless we assume a lower proton-to-electron ratio in NGC 253.

This work was supported by NASA grant NAG 5-2028 (Compton Gamma Ray Observatory Guest Investigator Program). This research has made use of the NASA/IPAC Extragalactic Database (NED), which is operated by the Jet Propulsion Laboratory, Caltech, under contract with the National Aeronautics and Space Administration. The authors thank M. Pohl for a careful reading of the text and for bringing our attention to the importance of energy losses to pion production.
Abraham, P. B., Brunstein, K. A.. \& Cline, T. L 1966, Phys. Rev., 150, 1088 Akyüz, A., Brouillet, N., \& Ozel, M. E. 1991, A\&A, 248, 419

Antonucci, R. R. J., \& Ulvestad, J. S. 1988, ApJ, 330, L9?

Beck, R., et al. 1979, A\&A, 77, 25

Beck, R., Carilli, C. L., Holdaway, M. A., \& Klein, U. 1994, A\&A, 292, 409

Bell, A. R. 1978a, MNRAS, 182, 147 .1978b, MNRAS, 182, 443

Bhattacharya, D., et al. 1994, ApJ 437, 173

Blandford, R. D., \& Ostriker, J. P. 1980, ApJ, 237, 793

Bloemen, H. 1989, ARA\&A, 27, 469

Canzian, B., Mundy, L. G., \& Scoville, N. Z. 1988, ApJ, 333, 157

Carilli, C. L., Holdaway, M. A., Ho, P. T. P., \& De Pree, C. G. 1992, ApJ 399, L59

Carlstrom, J. E. 1989, Ph.D. thesis, Univ. California, Berkeley

Carlstrom, J. E., Jackson, J., Ho, P. T. P., \& Turner, J. L. 1989, in The Interstellar Medium in External Galaxies, ed. D. J. Hollenbach \& H A. Thronson (Washington, DC: NASA), 337

Cavallo, G., \& Gould, R. J. 1971, Nuovo Cimento, 28, 77

Cox, P., \& Mezger, P. G. 1989, A\&A Rev., 1, 49

Dermer, C. D. 1986, A\&A 157,223

Drury, L. O'C., Markiewicz, W. J., \& Völk, H. J. 1989, A\&A, 225, 179

Elias, J. H., et al. 1978, ApJ, 220,25

Fichtel, C. E., et al. 1993, A\&AS, 97, 13

Ginzburg, V. L. \& Syrovatskii, S. I. 1964, The Origin of Cosmic Rays (New York: Macmillan)

Goldshmidt, O., \& Rephaeli, Y. 1995, ApJ, 444, 113

Heckman, T. M., Armus, L., \& Miley, G. K. 1990, ApJS, 74, 833

Helfer, T. T., \& Blitz, L. 1993, ApJ, 419, 86

Huang, Z. P., Thuan, T. X., Chevalier, R. A., Condon, J. J., \& Yin, Q. F. 1994, ApJ, 424, 114

Hughes, E. B., et al. 1980, IEEE Trans. Nucl. Sci., NS-27, 364

Hummel, E., Smith, P., \& van der Hulst, J. M. 1984, A\&A, 137, 138

Jackson, J. M., Paglione, T. A. D., Carlstrom, J. E., \& Rieu, N.-Q. 1995, ApJ, 438, 695

Kanbach, G., et al. 1988, Space Sci. Rev., 49,69 1989, in Proc. Gamma-Ray Observatory Sci. Workshop, ed. W. N. Johnson (Greenbelt : NASA), 2-1

Klein, U., \& Emerson, D. T. 1981, A\&A, 94, 29

Klein, U., Urbanik, M., Beck, R., \& Wielebinski, R. 1983, A\&A, 127, 177

Klein, U., Wielebinski, R., \& Morsi, H. W. 1988, A\&A, 190, 41

Kühr, H., Witzel, A., Pauliny-Toth, I. I. K., \& Nauber, U. 1981, A\&AS, 45, 367

\section{REFERENCES}

Longair, M. S. 1981, High Energy Astrophysics (Cambridge: Cambridge Univ. Press)

Mannheim, K., \& Schlickeiser, R. 1994, A\&A, 286, 983

Markiewicz, W. J., Drury, L. O'C., \& Völk, H. J. 1990, A\&A, 236, 487

Marscher, A. P., \& Brown, R. L. 1978, ApJ, 221, 588

Mauersberger, R., \& Henkel, C. 1989, A\&A, 223, 79

McCarthy, P. J., Heckman, T., \& van Breugel, W. 1987, AJ, 93, 264

Ormes, J., \& Freier, P. 1978, ApJ, 222, 471

Paglione, T. A. D., Jackson, J. M., Ishizuki, S., \& Rieu, N.-Q. 1995, AJ, 109, 1716

Piña, R. K., Jones, B., Puetter, R. C., \& Stein, W. A. 1992, ApJ, 401, L75

Pohl, M. 1994, A\&A, 287, 453

Reynolds, S. P., \& Ellison, D. C. 1992, ApJ, 399, L75

Rice, W., Lonsdale, C. J., Soifer, B. T., Neugebauer, G., Kopan, E. L. Lloyd, L. A., de Jong, T., \& Habing, H. J. 1988, ApJS, 68, 91

Rieke, G. H., Lebofsky, M. J., Thompson, R. I., Low, F. J., \& Tokunga, A. T. 1980, ApJ, 238, 24

Rieke, G. H., Lebolsky, M. J., \& Walker, C. E. 1988, ApJ, 325, 679

Rybicki, G. B., \& Lightman, A. P. 1979, Radiative Processes in Astrophysics (New York: John Wiley \& Sons)

Sage, L. M., Shore, S. N., \& Solomon, P. M. 1990, ApJ, 351, 422

Sanders, D. B., Solomon, P. M., \& Scoville, N. Z. 1984, ApJ, 276, 182

Seaquist, E. R., \& Odegard, N. 1991, ApJ, 369, 320

Sreekumar, P., et al. 1994, ApJ, 426, 105

Suchkov, A., Allen, R. J., \& Heckman, T. M. 1993, ApJ, 413, 542

Telesco, C. M., Campins, H., Joy, M., Dietz, K., \& Decher, R. 1991, ApJ, 369,135

Telesco, C. M., \& Harper, D. A. 1980, ApJ, 235, 392

Tammann, G. A. 1974, in Supernovae and Supernova Remnants, ed. C. B. Cosmovici (Dordrecht : Reidel), 155

Thompson, D. J., et al. 1993, ApJS, 86, 629

Ulvestad, J. S., \& Antonucci, R. R. J. 1991, AJ, 102, 875

- 1994, ApJ, 424, L29

Van Buren, D., \& Greenhouse, M. A. 1994, ApJ, 431, 640

Völk, H. J., Klien, U., \& Wielebinski, R. 1989a, A\&A, 237, 21 $1989 \mathrm{~b}, \mathrm{~A} \& \mathrm{~A}, 213, \mathrm{~L} 2$

Wall, W. F., Jaffe, D. T., Israel, F. P., \& Bash, F. N. 1991, ApJ, 380, 384

Wild, W., Harris, A. I., Eckart, A., Genzel, R., Graf, U. U., Jackson, J. M., Russeli, A. P. G., \& Stutzki, J. 1992, A\&A, 265, 447

Young, J. S., Xie, S., Kenney, J. D. P., \& Rice, W. L. 1989, ApJS, 70, 699 